\title{
Platelet volume indices: markers of carotid atherosclerosis in type 2 diabetes mellitus?
}

\section{ABSTRACT}

Background. Platelet volume indices (PVI) such as mean platelet volume (MPV), platelet distribution width (PDW), and platelet large cell ratio (P-LCR) are the indicators of platelet activity and may have a role in subclinical atherosclerosis and microvascular complications in type 2 diabetes mellitus (T2DM). We evaluated PVI in diabetics for their association with carotid intima media thickness (CIMT) and microvascular complications.

Methods. Participants - 105 T2DM patients and age, gender matched 105 controls were evaluated by history and complete blood counts (CBC) including PVI, blood sugars, $\mathrm{HbA}_{1 \mathrm{c}^{\prime}}$ lipid profile and microvascular complications. PVI were compared between cases and controls. Carotid Doppler was done and CIMT was correlated with PVI.

Results. PVI were found significantly higher in diabetic patients compared to controls. Mean MPV in cases vs. controls was $(11.09 \pm 1.02 \mathrm{fL}$ vs. $10.28 \pm 0.96 \mathrm{fL}, \mathrm{p} \leq$ 0.001), mean PDW (13.46 $\pm 1.96 \mathrm{fL}$ vs. $12.85 \pm 3.54$ $f L, p=0.12)$, mean P-LCR (31.92 $\pm 6.23 \%$ vs. $27.94 \pm$ $5.94 \%, p \leq 0.001)$. CIMT showed a positive significant association with MPV, PDW and PLCR, dyslipidemia and negative with glycemic control. PVI, especially MPV was significantly elevated in those with neuropathy, nephropathy and retinopathy.

Conclusion. PVI i.e. MPV, PDW, P-LCR are increased in diabetic patients. They correlate positively with CIMT,

Address for correspondence:

Rajashree Sanjay Khot, MD DNB (Medicine)

All India Institute of Medical Sciences

Nagpur, Plot no 2, Sector 20, Mihan, 441108 Nagpur, India

e-mail: rajashree.s.khot@gmail.com

Clinical Diabetology 2020, 9, 2, 103-111

DOI: $10.5603 /$ DK.2020.0008

Received: 13.10.2019 Accepted: 24.12.2019 implying cardiovascular risk. PVI have a positive association with microvascular complications also. PVI as determined by simple automated $\mathrm{CBC}$ can be used as markers of subclinical atherosclerosis and predictor of future cardiovascular events in T2DM. (Clin Diabetol 2020; 9; 2: 103-111)

Key words: T2DM, PVI, increased CIMT, microvascular complications

\section{Introduction}

In T2DM, platelets and their interaction with the vessel wall play a role in atherogenesis and in the formation of the coronary thrombus. Several haemorrheological alterations take place in both T1DM and T2DM. The erythrocytes are altered and become less deformable, contributing to increased whole blood viscosity. The platelet function is also altered in diabetes. Diabetic thrombocytopathy refers to differences in platelet function between diabetic and nondiabetic individuals. Among diabetic individuals, differences are due to the following: reduced membrane hydration, altered $\mathrm{Ca}^{2+}$ and $\mathrm{Mg}^{2+}$ homeostasis (increased intracellular $\mathrm{Ca}^{2+}$ mobilization and decreased intracellular $\mathrm{Mg}^{2+}$ ), increased arachidonic acid metabolism, increased TXA, synthesis, decreased prostacyclin production, decreased NO production, decreased antioxidant levels, increased expression of activation-dependent adhesion molecules (e.g., Gpllb-Illa, P-selectin) [1].

Platelets from patients with type 1 and type 2 diabetes exhibit enhanced platelet aggregation activity early in the disease course that may precede the development of cardiovascular disease (CVD). Numerous biochemical abnormalities have been found that correlate with platelet hyperreactivity. Platelets from diabetic patients exhibit reduced membrane hydration as described above, which may reflect changes in the 
lipid composition of the membrane or glycation of membrane proteins. Arachidonic acid metabolism is increased in platelets from diabetic patients; this leads to enhanced TXA 2 production and may contribute to increased platelet sensitivity. Platelets in type 2 diabetic individuals adhere to vascular endothelium and aggregate more readily than those in healthy people. Loss of sensitivity to the normal restraints exercised by prostacyclin [PGI (2)] and nitric oxide (NO) generated by the vascular endothelium presents as the major defect in platelet function. Insulin is a natural antagonist of platelet hyperactivity. It sensitizes the platelet to PGI (2) and enhances endothelial generation of PGI (2) and NO. Thus, the defects in insulin action in diabetes create a milieu of disordered platelet activity conducive to macrovascular and microvascular events [2]. Platelet size is also increased in diabetes. The large platelets having dense granules are more active biochemically, functionally, and metabolically, have higher thromboxane A2 levels, express more glycoprotein Ib and Ilb/Illa receptors, and could be a risk factor for developing coronary thrombosis [3]. Patients with type 2 diabetes mellitus, without proven vascular disease, exhibit platelet dysfunction and have increased platelet aggregation and aspirin insensitivity compared to non-diabetic patients with previous MI [4].

Many researchers have found an association between mean platelet volume, cardiovascular events like acute $\mathrm{Ml}$ and glycemic status. There are reports of negative association also. Hence, we wanted to explore the relation between T2DM and all platelet volume indices and whether these indices which are available as a part of complete blood counts as determined by automated counters, can be used as markers of future cardiovascular events.

The first structural change that can be detected in atherosclerosis is an increase in intima-media thickness (IMT). The value of CIMT as a surrogate endpoint in interventional or observational studies as an alternative to cardiovascular disease (CVD) is strengthened by its associations with risk factors and coronary or cerebrovascular events.

In this study, all components of platelet volume indices were compared with controls to see if they are altered in T2DM and if they are associated with increased CIMT and can be used as marker of subclinical atherosclerosis.

\section{Methods}

This hospital-based case control study was carried out in our tertiary care Centre from Januar 2016 to August 2017 after approval from institutional ethical committee.

\section{Study population}

105 consecutive patients of T2DM were selected from Diabetes Clinic and Medicine outpatient department. 105 healthy age and gender matched nondiabetic visitors or relatives, or unrelated attendants of hospitalized patients were selected as controls after written informed consent.

\section{Eligibility criteria}

Cases: T2DM in age $\geq 30$ years diagnosed by ADA criteria.

Patients were excluded if they had diabetes other than T2DM, any acute complications of diabetes, acute infections, critical illness, myeloproliferative disorders, thrombocytopenia due to any other cause e.g. immune thrombocytopenia, hemoglobin $<10 \mathrm{~g} / \mathrm{dL}$ in females and $<11 \mathrm{~g} / \mathrm{dL}$ in males, pre-existing peripheral vascular disease, stroke and/or angiographically proven coronary artery disease (CAD) with or without revascularization, or on antiplatelet drugs the controls were age and sex matched healthy subjects. Exclusion criteria for controls were same as those for cases.

Detailed diabetic history with respect to onset, duration, treatment and hypoglycaemic episodes was obtained. Relevant history, personal history, any addictions or drug abuse and socioeconomic data was recorded. Cardiovascular risk factors were evaluated in all patients according to standard definitions. *Smokers included current smokers and ex-smokers of $<12$ months, whereas non-smokers included ex-smokers of more than 12 months and never smokers. **Alcoholism was defined as more than 14 drinks per week in men and more than 7 drinks in women. ${ }^{* *}$ Hypertensives included patients on antihypertensive medications or newly detected hypertension as per JNC VIII criteria. \#Positive family history was defined as occurrence of CAD or sudden cardiac death in first degree relatives before 40 years of age. \#\#Dyslipidemia was defined as serum ( $\mathrm{Sr}$ ) cholesterol $>200 \mathrm{mg} / \mathrm{dL}$ and/or serum triglycerides $>150 \mathrm{mg} / \mathrm{dL}$ and/or serum $\mathrm{HDL}<40 \mathrm{mg} / \mathrm{dL}$ in males and $<50 \mathrm{mg} / \mathrm{dL}$ in females (Table 1).

Physical measurements specifically height, weight, BMI, were recorded and blood pressure measurements, electrocardiogram were done in each patient.

All patients were evaluated for macro and microvascular complications of diabetes. Those with macrovascular complications were excluded. Evaluation for microvascular complications was done as follows - peripheral neuropathy: assessed by Toronto Clinical neuropathy score and SW monofilament test. Diabetic retinopathy was assessed by fundus examination through dilated pupil by ophthalmologist and diabetic nephropathy was assessed by microalbuminuria and eGFR. 
Table 1. Baseline characteristics, cardiovascular risk factors and PVI in cases and controls

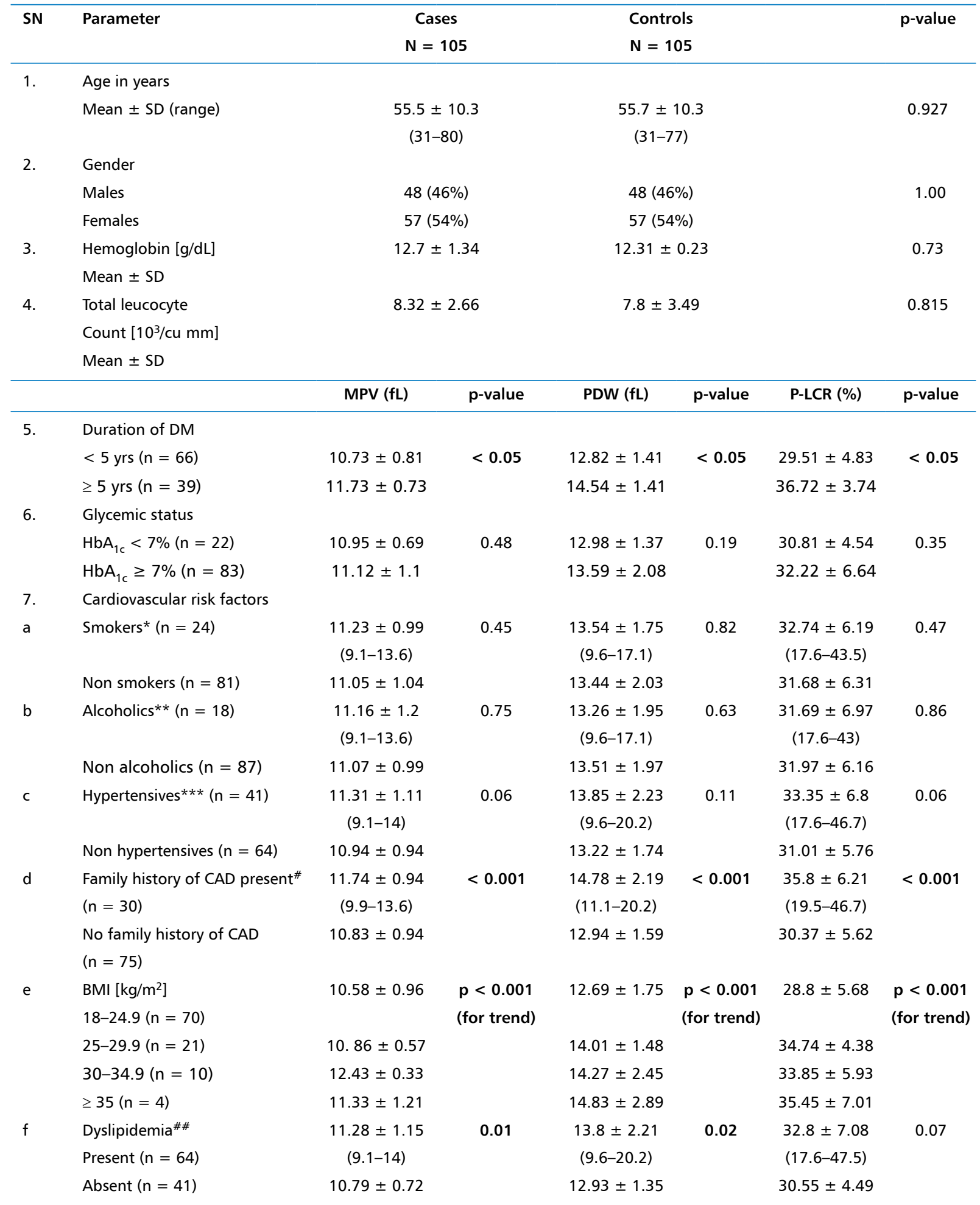

MPV — mean platelet volume; PDW - platelet distribution width; P-LCR — platelet large cell ratio; DM — diabetes mellitus; BMI — body mass index

$C B C$, fasting and post-meal blood sugar, lipid profile, microalbuminuria, Sr creatinine, eGFR by Cockcroft Gault formula and glycosylated haemoglobin $\left(\mathrm{HbA}_{1 \mathrm{c}}\right)$ by standardized HPLC method were performed in all cases. In controls CBC for PVI and fasting, post meal blood sugar and $\mathrm{HbA}_{1 \mathrm{C}}$ and other relevant investigations were done.

Platelet volume indices [5] - the PVI are universally available with routine blood counts by automated 
hemograms. Following PVI were studied in cases and controls.

Platelet count: Platelet counts are laboratory measure of concentration of platelets in blood. The standard ranges from 1.5 to $4.5 \times 10^{6} / \mathrm{cu} \mathrm{mm}$.

Mean platelet volume (MPV): This parameter provides a statement on the MPV between the lower discriminator and the upper discriminator. The standard ranges from 8 to $12 \mathrm{fL}$.

Platelet distribution width (PDW): PDW indicates the platelet distribution width measured at $20 \%$ relative height of the total height of the curve. An increased PDW is an indicator for the anisocytosis of platelets. Standard PDW ranges from 9 to $14 \mathrm{fL}$.

Platelet large cell ratio (P-LCR): P-LCR indicates the percentage of large platelets with a volume $>$ $12 \mathrm{fL}$ and is presented in \%. The standard range is $15-35 \%$. An increase may be an indication for platelet aggregates, micro erythrocytes and giant platelets also regenerating large platelets.

Plateletcrit (Pct): Plateletcrit is equivalent to the sum of platelet impulses which are individually detected by means of the impedance measurement principle and thus it is the platelet equivalent to the hematocrit of the RBCs. The standard ranges from 0.22 to $0.24 \%$.

Carotid intima medial thickness: Carotid Doppler was done by single expert sonologist in all cases evaluating both the carotid arteries. Thickening of the intima-media greater than $0.8 \mathrm{~mm}$ was considered as abnormal and represented the earliest change of atherosclerosis. Cut-off was based on findings of screening of normal persons and patients with subclinical atherosclerosis prior to this study at our institute by qualified sonologist. Carotid plaques and percentage carotid stenosis were also determined. The far wall of the common carotid artery 1-2 cm proximal of carotid bulb was used for measurement. The leading edge of vascular lumen-intima was selected as the internal measurement site and the leading edge of the mediaadventitia as the external limit. The measurement was repeated twice at the same site and then performed in the same way three times at the corresponding opposite site. Mean of CIMT of three sites of a side was taken for calculation of CIMT of that side.

\section{Statistical analysis}

Collected data was entered in MS-Excel 2010 and corrected for typographic errors and analyzed using SPSS 16.0 version. Descriptive statistics like proportions, mean and standard deviation was used for continuous variables. For categorical variables, chi-square test was used. To decipher independent association of PVI with CIMT, multiple logistic regression analysis was done with CIMT as the dichotomous outcome variable (dependent variable). Independent T test (unpaired t test) was used to compare between two groups and ANOVA was used to compare the continuous variables among more than two groups. The confidence limit for significance was fixed at $95 \%$ level with $p$-value $<0.05$.

\section{Results}

It was observed that out of 105 cases and controls, $48(46 \%)$ were males and 57 (54\%) were females with a M:F ratio 1:1.2. Most cases (34\%) and controls (32\%) were in the age group of 51-60 years. In controls, as the age advanced, mean of all platelet indices increased and the difference was statistically significant. The MPV increased from $10.8 \pm 0.91$ in 32-40 years age group to $11.7 \pm 0.82$ in $71-80$ years age group $(p=0.01)$. This was not observed in diabetics. This could be due to baseline increase in MPV, PDW, P-LCR in diabetics due to disease itself. Hence the age-related increment did not occur. There was no significant difference in mean MPV, PDW and P-LCR based on gender.

Most patients (59\%) had duration of diabetes of more than 5 years, $11.4 \%$ cases were newly diagnosed and $11.4 \%$ had diabetes of more than 10 years. The mean PVI gradually increased with duration of diabetes up to10 years and were significant for MPV, PDW and P-LCR. Beyond 10 years, PVI were not much altered. This could be explained as number of patients with duration greater than 10 years were much less $(11 \%)$ and the disease related changes in these platelet indices had already occurred and may have reached a plateau (Table 1).

\section{PVI in T2DM as compared to healthy controls}

It was observed that all the platelet indices except platelet count were increased in diabetics as compared to healthy controls. $16 \%$ of diabetics had increased MPV, 34\% had increased PDW, 26\% had increased P-LCR and $76 \%$ had increased plateletcrit as compared to $1 \%, 15 \%, 23 \%, 63 \%$ controls respectively. Platelet count was within normal range. Mean of MPV, PDW, $\mathrm{P}-\mathrm{LCR}$ and plateletcrit were also increased in cases as compared to controls, however statistically significant increase was observed only in MPV and P-LCR (Table 2).

\section{Cardiovascular risk factors and PVI}

Dyslipidemia (61\%) (increased cholesterol and triglycerides and decreased $\mathrm{HDL}$ ) was the most common cardiovascular risk factor in diabetics followed by hypertension (40\%), BMI $\geq 25 \mathrm{~kg} / \mathrm{m}^{2}$ (34\%) and family history of IHD (28.6\%).

Mean MPV, PDW were significantly increased in patients with hypercholesterolemia, and mean MPV, PDW 
Table 2. Platelet volume indices (PVI) in T2DM and nondiabetics

\begin{tabular}{lccc}
\hline PVI & $\begin{array}{c}\text { Cases (T2DM) } \\
(\mathbf{n}=105)\end{array}$ & $\begin{array}{c}\text { Controls } \\
\text { (nondiabetics) } \\
(\mathbf{n}=105)\end{array}$ & p-value \\
& & $10.28 \pm 0.96$ & $<0.001$ \\
\hline MPV (fL) & $11.09 \pm 1.02$ & $(6-12.1)$ & \\
& $(8.2-14)$ & $12.85 \pm 3.54$ & 0.12 \\
PDW (fL) & $13.46 \pm 1.96$ & $(4.2-38.6)$ & \\
& $(9.4-20.2)$ & $27.94 \pm 5.94$ & $<0.001$ \\
P-LCR (\%) & $31.92 \pm 6.23$ & $(14.5-39.5)$ & \\
& $(17.6-47.5)$ & $2.82 \pm 0.69$ & 0.14 \\
Platelet count & $2.69 \pm 0.55$ & $(1.5-4.57)$ & \\
(10\%/cu mm) & $(1.63-4.26)$ & $0.28 \pm 0.07$ & 0.18 \\
Plateletcrit (\%) & $0.29 \pm 0.05$ & 0.28 & \\
& $(0.18-0.47)$ & $(0.13-0.5)$ & \\
& & &
\end{tabular}

MPV - mean platelet volume; T2DM - type 2 diabetes mellitus; PDW - platelet distribution width; P-LCR - platelet large cell ratio

and P-LCR were significantly increased in patients with hypertriglyceridemia. Mean MPV, PDW and P-LCR were significantly increased in female patients with HDL less than $50 \mathrm{mg} / \mathrm{dl}$ as compared to those with normal levels.

PVI like MPV, PDW and P-LCR were significantly increased in cases with $\mathrm{BMI} \geq 25$ and family history of IHD. There was no significant association of PVI with CV risk factors like hypertension, alcoholism and smoking (Table 1).

\section{Carotid atherosclerosis and platelet volume indices}

All diabetics underwent carotid doppler and it was observed that on right side, $44 \%$ patients had CIMT < $0.8 \mathrm{~mm}, 55 \%$ had CIMT $\geq 0.8 \mathrm{~mm}$. On left side, $46 \%$ had CIMT $<0.8 \mathrm{~mm}, 53 \%$ had CIMT $\geq 0.8 \mathrm{~mm}$. The mean CIMT of both sides were comparable $(0.78 \pm$ $0.22 \mathrm{~mm}$ on right and $0.79 \pm 0.23 \mathrm{~mm}$ on left). On right side, $4.7 \%$ cases had $<50 \%$ stenosis and exactly equal cases had $\geq 50 \%$ stenosis, whereas on left, $7.6 \%$ cases had $<50 \%$ stenosis and no patient had $\geq 50 \%$ stenosis. Plaques without significant occlusion were detected on right side in $20.9 \%$ and on left side in $8.5 \%$ cases. When PVI were correlated with CIMT it was observed that mean MPV, PDW, P-LCR were significantly increased, plateletcrit was marginally increased and mean platelet count was decreased in patients with CIMT $\geq 0.8$ (Table 3).

When mean CIMT was plotted against these PVI, it was observed that MPV, PDW and P-LCR had moderate positive linear relationship which was statistically significant. Platelet count had weak negative linear re-
Table 3. Association of platelet volume indices (PVI) with carotid intima media thickness (CIMT)

\begin{tabular}{lccc}
\hline $\begin{array}{l}\text { PVI } \\
\text { Mean + SD } \\
\text { (range) }\end{array}$ & $\begin{array}{c}\text { CIMT }<0.8 \\
(\mathbf{n}=49)\end{array}$ & $\begin{array}{c}\text { CIMT } \geq 0.8 \\
(\mathbf{n}=56)\end{array}$ & p-value \\
\hline MPV (fL) & $10.57 \pm 0.83$ & $11.53 \pm 0.97$ & $<0.001$ \\
& $(8.2-12.8)$ & $(9.1-14)$ & \\
PDW (fL) & $12.53 \pm 1.39$ & $14.28 \pm 2.03$ & $<0.001$ \\
& $(9.4-16.1)$ & $(9.6-20.2)$ & \\
P-LCR (\%) & $28.66 \pm 4.78$ & $34.76 \pm 6.06$ & $<0.001$ \\
& $(19.3-42.5)$ & $(17.6-47.5)$ & \\
Platelet count & $2.75 \pm 0.60$ & $2.65 \pm 0.51$ & 0.37 \\
(10\%/cu mm) & $(1.75-4.26)$ & $(1.63-3.52)$ & \\
Plateletcrit $(\%)$ & $0.27 \pm 0.06$ & $0.29 \pm 0.054$ & 0.14 \\
& $(0.18-0.47)$ & $(0.19-0.4)$ & \\
& & & \\
& & &
\end{tabular}

MPV - mean platelet volume; PDW - platelet distribution width; P-LCR - platelet large cell ratio

lationship ( $r=-0.231)$ and was statistically significant. However, plateletcrit had no relationship with CIMT.

Multiple logistic regression analysis with CIMT as dichotomous dependent outcome variable and significant cardiovascular risk factors as independent predictor variable

MPV was significantly associated and had positive co-relation with age $(r=0.335, p<0.001)$, duration of DM ( $r=0.437, p<0.001)$, BMI $(r=0.268, p=$ $0.006)$, cholesterol $(r=0.297, p=0.002)$, triglyceride $(r=0.204, p=0.03)$, LDL-C $(r=0.222, p=0.02)$ and CIMT ( $r=0.638, p<0.001)$. MPV was significantly but negatively co-related to HDL-C $(r=-0.307, p<0.001)$. No association existed between MPV and $\mathrm{HbA}_{1 \mathrm{c}}$.

On MLR analysis, when platelet indices were assessed as independent risk factors after controlling for BMI, dyslipidemia and positive family history of $I H D$, they were not significant. On univariate analysis, MPV, PDW and P-LCR were significantly associated with increased CIMT. Hence it can be concluded that MPV, PDW and P-LCR are not independent cardiovascular risk factors but may have an effect in association with conventional risk factors, leading to subclinical atherosclerosis as determined by increased CIMT (Figure 1).

\section{Association of PVI with microvascular complications of T2DM}

Mean MPV, PDW and P-LCR were significantly higher, and mean platelet count was significantly lower in patients with retinopathy as compared to those without retinopathy. Mean MPV, PDW and P-LCR were signifi- 


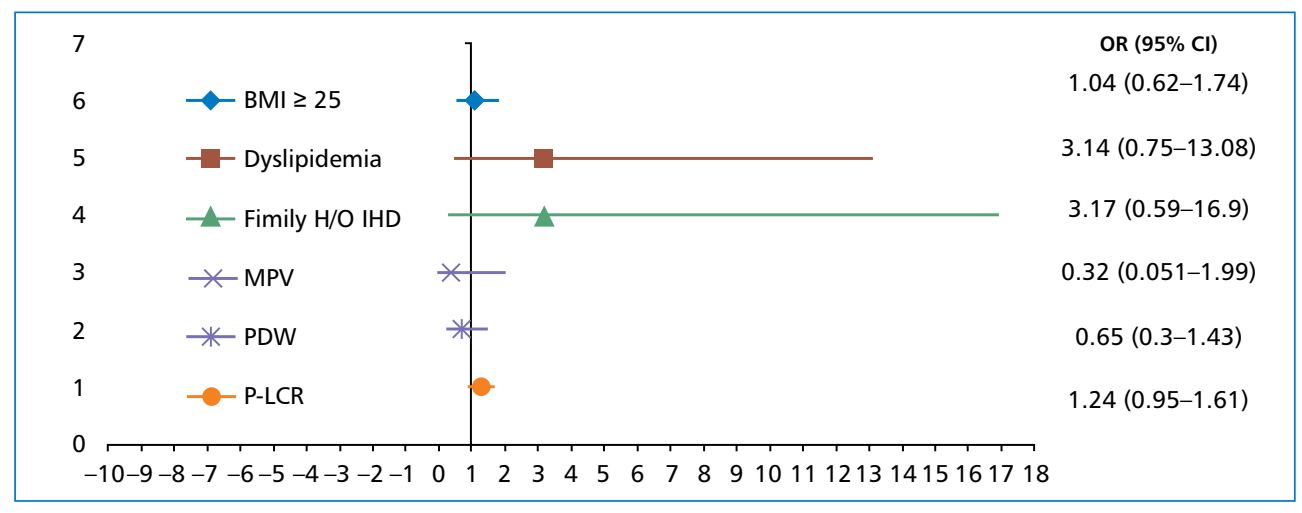

Figure 1. Multiple logistic regressions with CIMT as dichotomous dependent outcome variable. MPV — mean platelet volume; PDW - platelet distribution width; P-LCR - platelet large cell ratio

Table 4. Association of platelet volume indices (PVI) with microvascular complications

\begin{tabular}{|c|c|c|c|c|c|c|}
\hline Complication & MPV (fL) & p-value & PDW (fL) & p-value & P-LCR (\%) & $p$-value \\
\hline Retinopathy present $(n=29)$ & $\begin{array}{c}12.09 \pm 0.88 \\
(10.3-14)\end{array}$ & $<0.001$ & $\begin{array}{c}15.33 \pm 1.94 \\
(12-20.2)\end{array}$ & $<0.001$ & $\begin{array}{c}37.83 \pm 5.09 \\
(25.1-47.5)\end{array}$ & $<0.001$ \\
\hline Retinopathy absent $(\mathrm{n}=76)$ & $\begin{array}{c}10.7 \pm 0.79 \\
(8.2-12.5)\end{array}$ & & $\begin{array}{c}12.75 \pm 1.44 \\
(9.4-16.1)\end{array}$ & & $\begin{array}{c}29.67 \pm 5.12 \\
(17.6-42.5)\end{array}$ & \\
\hline Neuropathy present $(n=27)$ & $\begin{array}{c}11.86 \pm 0.88 \\
(9.7-13.6)\end{array}$ & $<0.001$ & $\begin{array}{c}14.72 \pm 1.74 \\
(11-19.9)\end{array}$ & $<0.001$ & $\begin{array}{c}36.26 \pm 5.44 \\
(22.8-47.5)\end{array}$ & $<0.001$ \\
\hline Neuropathy absent $(n=78)$ & $\begin{array}{c}10.82 \pm 0.93 \\
(8.2-14)\end{array}$ & & $\begin{array}{c}13.03 \pm 1.85 \\
(9.4-20.2)\end{array}$ & & $\begin{array}{c}30.42 \pm 5.85 \\
(17.6-46.7)\end{array}$ & \\
\hline Nephropathy present $(n=55)$ & $\begin{array}{c}11.54 \pm 1.03 \\
(9.2-14)\end{array}$ & $<0.001$ & $\begin{array}{c}14.33 \pm 2.03 \\
(11-20.2)\end{array}$ & $<0.001$ & $\begin{array}{l}34.7 \pm 6.27 \\
(22.8-47.5)\end{array}$ & $<0.001$ \\
\hline Nephropathy absent $(n=50)$ & $\begin{array}{c}10.58 \pm 0.75 \\
(8.2-12.4)\end{array}$ & & $\begin{array}{c}12.51 \pm 1.36 \\
(9.4-14.8)\end{array}$ & & $\begin{array}{l}28.8 \pm 4.67 \\
(17.6-38.2)\end{array}$ & \\
\hline
\end{tabular}

MPV — mean platelet volume; PDW — platelet distribution width; P-LCR — platelet large cell ratio

cantly higher, and mean platelet count was lower but non-significantly in patients with neuropathy as compared to those without neuropathy. Mean MPV, PDW and P-LCR were significantly higher, and mean platelet count was significantly lower in patients with nephropathy as compared to those without nephropathy (Table 4).

\section{Discussion}

Diabetes is considered as a 'prothrombotic' state. Platelet activation is an important factor, the determinants of which are platelet volume indices i.e. mean plate volume (MPV), platelet distribution width (PDW) and platelet large cell ratio (P-LCR). When we compared the PVI in T2DM with healthy controls we observed that MPV, P-LCR were significantly increased in diabetics. PDW and plateletcrit were increased but statistically insignificant and platelet counts were unaltered. In diabetes, there is increased thrombopoiesis and circulation of large, young and activated platelets which undergo more frequent episodes of release of granules, accelerated sequestration in the circulation and have reduced survival, thus platelet count is decreased. As plateletcrit is the volume occupied by platelets in the blood as a percentage, and directly related to platelet count, both the indices decrease in diabetics. However, no such decrease in plateletcrit was seen in our study. Factors that contribute directly to greater platelet reactivity in diabetics include metabolic abnormalities such as hyperglycemia and hyperlipidemia, both insulin resistance (relative insulin deficiency) and absolute insulin deficiency, as well as associated conditions such as oxidative stress, inflammation, and endothelial dysfunction [6].

Many studies have reported increase in MPV in diabetics as compared to non-diabetics. Our results were in accordance to studies done by Kodiatte et al. [7], Papanas et al. [8], and Alhadas et al. [9]. Hekimsoy et al. [10] also reported increased MPV in diabetics but 
reported low platelet counts in diabetics. We did not observe any alteration in platelet counts in diabetics. MPV is an indicator of the average size and activity of platelets. Larger platelets are younger, more reactive and aggregable. Hence, they contain denser granules, secrete more serotonin and $\beta$-thromboglobulin, and produce more thromboxane $\mathrm{A} 2$ than smaller platelets. MPV reflects the state of thrombogenesis. There might be small bleeds due to the rupture of atherothrombotic plaques leading to increased platelet recruitment, hyper reactivity, and bone marrow stimulation. Thus, high MPV is emerging as a new risk factor for the vascular complications of DM of which atherothrombosis plays a major role [7]. Many researchers have observed an association of increased MPV with cardiovascular events like myocardial infarction and acute coronary syndrome. We did find an increased PDW in diabetics, but the difference was statistically insignificant. Contrary to this Jindal et al. [11] observed that PDW was significantly higher in diabetics. It could be due to larger platelets being hyper-reactive produce more prothrombotic factors. Platelet activation causes changes in platelet morphology and formation of pseudopodia. The enlarged platelets with lots of pseudopodia differ in the size, possibly affecting the platelet distribution. Our study also observed statistically significant increase in P-LCR which implied that platelet size was larger in diabetics. This finding has been corroborated by many studies.

Studies have shown that platelet count and size might be gender and age dependent. Advanced age was directly associated with increased MPV as concluded in a study by Lippie et al. [12], where they attributed this increase in platelet volume to other cardiovascular risk factors like hypertension, dyslipidemia etc. that occur with advancing age. We observed that MPV, PDW and P-LCR also increased significantly with age in controls, but not in diabetics. There was no significant association of platelet count and plateletcrit with age.

It was observed that platelet count and plateletcrit was increased significantly in females as compared to males. It was in accordance with a study by Bain et al. [13]. The difference can possibly be ascribed to the different hormonal profiles amongst the gender or the effect of menstrual cycle on the haemostatic mechanism as thrombocytosis is associated with bleeding. There were no significant differences among the genders with respect to MPV, PDW or P-LCR.

When conventional cardiovascular risk factors were studied, it was observed that, dyslipidemia (61\%) was the most common risk factor in diabetics followed by hypertension (40\%), BMI $\geq 25 \mathrm{~kg} / \mathrm{m}^{2}$ (34\%) and family history of CAD (28.6\%). In controls, dyslipidemia (28\%) was the most common risk factor followed by hypertension (27\%), smoking (19\%), and family history of CAD (17\%). PVI were significantly higher in diabetics with dyslipidemia (MPV and PDW), family history of CAD (MPV, PDW and P-LCR) and BMI $\geq 25 \mathrm{~kg} / \mathrm{m}^{2}$ (MPV, PDW and P-LCR). PVI were high in patients with hypertension and smoking but statistically not significant. Nechita et al. [14] also observed that patients with cardiovascular risk factors, especially complex ones like the metabolic syndrome had an increased MPV, as did the patients with unstable angina whether associated with the risk factors. They implied that MPV could be used to differentiate unstable angina from non-cardiac chest pain. In a study by Swaminathan et al. [15] MPV was found to be higher in subjects with type 2 diabetes and significantly increased in diabetics with poor glycemic control and having a longer duration of diabetes. In contrast to most studies we did not find a significant association between PVI and glycemic control [16].

\section{PVI and carotid intima media thickness}

Carotid doppler was performed in all diabetics and patients were divided in 2 groups based on CIMT; CIMT $<0.8$ and CIMT $\geq 0.8 \mathrm{~mm}$. PVI were compared between 2 groups. It was observed that mean MPV, PDW, P-LCR were increased significantly in patients with $\mathrm{CIMT} \geq 0.8$. Mean platelet count was decreased and plateletcrit was increased but statistically insignificant.

In diabetic patients, hyperglycemia impairs vascular endothelial cells and NO production which contributes to the formation of atherosclerosis by mediating leukocyte activation and adhesion to the endothelium via platelet P-selection [17]. As the platelets participate in the formation of atherosclerotic plaque, they are consumed and platelet count may decrease with or after the development of atherosclerosis. Our study revealed that platelet count has weak negative linear relationship with CIMT ( $r=-0.231)$ which was statistically significant. Also, other indices like MPV, PDW and P-LCR had moderate positive linear relationship with CIMT which was statistically significant. However, plateletcrit had no relationship with CIMT.

Many efforts have focused on the elucidation of common pathophysiological mechanisms in obesity, type 2 diabetes and atherosclerosis, supporting the role played by inflammation. A feature of inflammatory activity is the increase in circulating plasma of acutephase proteins produced by the liver such as C-reactive protein (CRP) and fibrinogen. Fibrinogen levels have been shown to be associated with enhanced platelet aggregation and smooth muscle cell proliferation. Furthermore, there is a strong association of fibrino- 
gen with blood viscosity and thrombus formation and circulating levels of fibrinogen have been known to have a strong and consistent relationship with CAD [18]. However, in our study we did not estimate the hsC-reactive protein or fibrinogen levels, and this is a limitation of this study which may have overemphasized the role of platelet indices.

According to Wan et al. [19] MPV had a high positive correlation with IMT $(p<0.001)$. Adam et al. [20] in their correlation analysis showed a positive association between PDW and the degree of carotid stenosis. However, they did not find a significant correlation between carotid stenosis and MPV. According to study by Yilmaz et al. [21] the calcified plaque group was compared with the non-calcified plaque group in terms of MPV and PDW. The findings of their study indicated that the non-calcified plaques that cause intermediate carotid artery stenosis are associated with a significantly higher MPV value compared to the calcified plaques. There was no difference between the two groups with respect to PDW values. However, study by Kim et al. [22] found that MPV was strongly associated with the severity of glycemic control but not significantly associated with the early and late stages of atherosclerotic vascular changes in type 2 diabetes mellitus patients. They concluded that MPV is not a reliable marker for subclinical atherosclerosis in a diabetic population. This was possibly confounded by the close association of MPV with poor glycemic control.

Thoracic aortic intima-media thickness (IMT) was reported as an earlier marker of preclinical atherosclerosis than carotid IMT. Yüksel Kalkan et al. [23] reported that MPV is independently related to the extent of subclinical thoracic aortic atherosclerosis. Thus increases in MPV may be a crucial biochemical marker for initial atherosclerosis.

On multiple logistic regression analysis with CIMT as dichotomous dependent outcome variable and significant cardiovascular risk factors as independent variables, it was observed that MPV, PDW and P-LCR were not independent risk factors for cardiovascular disease, but had an effect in association with conventional risk factors, leading to subclinical atherosclerosis as determined by increased CIMT, although on univariate analysis, these PVI were significantly associated with increased CIMT.

If vascular damage was only due to increased number of large and reactive platelets, then the rate of damage would have been constant for the duration of disease and independent of diabetic control. This clearly shows that platelet reactivity alone cannot explain the progression of vascular complications in DM since there are other vascular risk factors that may be influenced by degree of control of diabetes. Platelet number and reactivity along with cardiovascular co-morbidities such as hypertension, albuminuria, obesity, cigarette smoking, and dyslipidemia also contribute to progression of diabetes and its effect on PVI. Thus, it shows that there are other factors which may account for the thrombotic potential of diabetics.

According to Alvitigala et al. [24] and Majumdar et al. [25] PVI have the potential to be used as a preliminary test to identify high-risk patients for myocardial infarction along with other supportive clinical investigations.

\section{PVI and microvascular complications}

Studies have found an association between increased MPV and CAD but very few studies have explored the relation between PVI and all the microvascular complications. In our study, diabetic nephropathy $(82 \%)$ was the most common microvascular complication, followed by retinopathy (46\%), and neuropathy (46\%). MPV, PDW and P-LCR were significantly higher in diabetics with microvascular complications. Platelet count was significantly increased in retinopathy and nephropathy but insignificantly in neuropathy. Papanas et al. [8] found that MPV was higher in patients with microvascular complications. Ates et al. [26] proved an association between degree of retinopathy and mean MPV. Buch et al. [27] and Alhadas et al. [9] concluded that MPV and PDW were significantly associated with microvascular complications. Jindal et al. [11], found that only PDW was significantly higher in diabetics with complications. This suggests a role of the enhanced platelet activity in the pathogenesis of microvascular complications.

Thus, PVI namely MPV, PDW, P-LCR are increased in diabetics and have a positive association with increased CIMT. They also correlate with all $3 \mathrm{mi}-$ crovascular complications of diabetes. They are not independent predictors but may be useful marker of carotid atherosclerosis in association with other cardiovascular risk factors.

\section{Limitations of the study}

Time bound study with relatively small sample size. Many confounding factors for poor glycemic control and increased complications in our subset of patients may have overestimated the association of platelet volume indices with microvascular complications. Also, we could not estimate hs CRP and fibrinogen levels which are also surrogate markers of inflammation associated with atherosclerosis. 


\section{Acknowledgements}

We are grateful to The Dean, Indira Gandhi Govt Medical College, Nagpur for permitting us and supporting us to carry out this study.

\section{Conflict of interest}

The authors declare to have no conflict of interest.

Contributors: RW conceived the study, reviewed the literature, acquired the data, analyzed it and drafted the article. RSK formulated the study design, helped with informed consent of the patients and investigations, and edited the article. PPJ helped refining the study design and helped in preparation of final version of the article.

Funding: self, and institute

\section{Patient consent for publication: not required}

Ethics approval: the study was approved by the Institutional Ethics committee, Indira Gandhi Govt Medical College, Nagpur

\section{REFERENCES}

1. Ghoshal K, Bhattacharyya M. Overview of platelet physiology: its hemostatic and nonhemostatic role in disease pathogenesis. Scientific World Journal. 2014: 781857, doi: 10.1155/2014/781857, indexed in Pubmed: 24729754.

2. Vinik Al, Erbas T, Park TS, et al. Platelet dysfunction in type 2 diabetes. Diabetes Care. 2001; 24(8): 1476-1485, doi: 10.2337/ diacare.24.8.1476, indexed in Pubmed: 11473089.

3. Giles H, Smith RE, Martin JF. Platelet glycoprotein IIb-IIla and size are increased in acute myocardial infarction. Eur J Clin Invest. 1994; 24(1): 69-72, doi: 10.1111/j.1365-2362.1994.tb02062.x, indexed in Pubmed: 8187810.

4. Mylotte D, Kavanagh GF, Peace AJ, et al. Platelet reactivity in type 2 diabetes mellitus: a comparative analysis with survivors of myocardial infarction and the role of glycaemic control. Platelets. 2012; 23(6): 439-446, doi: 10.3109/09537104.2011.634932, indexed in Pubmed: 22150374.

5. Wiwanitkit V. Plateletcrit, mean platelet volume, platelet distribution width: its expected values and correlation with parallel red blood cell parameters. Clin Appl Thromb Hemost. 2004; 10(2): 175-178.

6. Schneider DJ. Factors contributing to increased platelet reactivity in people with diabetes. Diabetes Care. 2009; 32(4): 525-527, doi: 10.2337/dc08-1865, indexed in Pubmed: 19336636.

7. Kodiatte TA, Manikyam UK, Rao SB, et al. Mean platelet volume in type 2 diabetes mellitus. J Lab Physicians. 2012; 4(1): 5-9, doi: 10.4103/0974-2727.98662, indexed in Pubmed: 22923915.

8. Papanas N, Symeonidis G, Maltezos E, et al. Mean platelet volume in patients with type 2 diabetes mellitus. Platelets. 2004; 15(8): 475-478, doi: 10.1080/0953710042000267707, indexed in Pubmed: 15763888.

9. Alhadas K, Santos S, Freitas M, et al. Are platelet indices useful in the evaluation of type 2 diabetic patients? Jornal Brasileiro de Patologia e Medicina Laboratorial. 2016; 52(2): 96-102, doi: 10.5935/1676-2444.20160017.
10. Hekimsoy Z, Payzin B, Ornek T, et al. Mean platelet volume in type 2 diabetic patients. J Diabetes Complications. 2004; 18(3): 173-176, doi: 10.1016/S1056-8727(02)00282-9, indexed in Pubmed: 15145330.

11. Jindal S, Gupta S, Gupta R, et al. Platelet indices in diabetes mellitus: indicators of diabetic microvascular complications. Hematology. 2011; 16(2): 86-89, doi: 10.1179/102453311X129 02908412110, indexed in Pubmed: 21418738.

12. Lippi G, Meschi T, Borghi L. Mean platelet volume increases with aging in a large population study. Thromb Res. 2012; 129(4): e159-e160, doi: 10.1016/j.thromres.2011.12.031, indexed in Pubmed: 22261476.

13. Bain BJ. Platelet count and platelet size in males and females. Scand J Haematol. 1985; 35(1): 77-79, doi: 10.1111/j.16000609.1985.tb00804.x, indexed in Pubmed: 4048863.

14. Nechita A, Delcea C, Enache V, et al. Metabolic syndrome and mean platelet volume variation in patients with chest pain and negative cardiac enzymes. J Med Life. 2013; 6(2): 156-160, indexed in Pubmed: 23904875.

15. Swaminathan A, Amitkumar K, Ganapathy S, et al. Evaluation of mean platelet volume and other platelet parameters in subjects with Type-2 diabetes mellitus. Natl J Physiol Pharm Pharmacol. 2017; 7(1): 51-54, doi: 10.5455/njppp.2016.6.0719518072016.

16. ManojSaluja, yogesh kumar swami, SR meena. Study of impact of glycemic status $(\mathrm{HbA} 1 \mathrm{c})$ on platelet activity measured by mean platelet volume \& vascular complications in diabetics. JAPI. 2019; 67: 26-29.

17. Ferreiro JL, Gómez-Hospital JA, Angiolillo DJ. Platelet abnormalities in diabetes mellitus. Diab Vasc Dis Res. 2010; 7(4): 251-259, doi: 10.1177/1479164110383994, indexed in Pubmed: 20921090.

18. Sarangi R, Mahapatra S, Padhi S. Association of plasma fibrinogen and serum high-sensitivity C-reactive protein in type 2 diabetes mellitus. J Curr Res Sci Medi. 2015: 21-26.

19. Wan H. Zhang L, Yi T, Zhang L. The relationship between the level of mean platelet volume and the carotid artery intima-media thickness in patients with type 2 diabetes mellitus. Int J Diabetes Dev Ctries. 2017; 37(2): 137-140.

20. Adam G, Kocak E, Reşorlu M, et al. Evaluation of platelet distribution width and mean platelet volume in patients with carotid artery stenosis. Angiology. 2015; 66(4): 375-378, doi: 10.1177/0003319714548682, indexed in Pubmed: 25313243.

21. Yılmaz F, Köklü E, Kizilirmak Yilmaz F, et al. Evaluation of mean platelet volume and platelet distribution width in patients with asymptomatic intermediate carotid artery plaque. Kardiol Pol. 2017; 75(1): 35-41, doi: 10.5603/KP.a2016.0129, indexed in Pubmed: 27714714.

22. Kim ES, Mo EY, Moon SD, et al. Mean platelet volume is closely associated with serum glucose level but not with arterial stiffness and carotid atherosclerosis in patients with type 2 diabetes. J Clin Endocrinol Metab. 2015; 100(9): 3502-3508, doi: 10.1210/ JC.2015-1540, indexed in Pubmed: 26120789.

23. Yüksel Kalkan G, Gür M, Baykan AO, et al. Mean platelet volume is associated with aortic intima-media thickness in patients without clinical manifestation of atherosclerotic cardiovascular disease. Anatol J Cardiol. 2015; 15(9): 753-758, doi: 10.5152/ akd.2014.5576, indexed in Pubmed: 25592097.

24. Alvitigala BY, Azra MA, Kottahachchi DU, et al. A study of association between platelet volume indices and ST elevation myocardial infarction. Int J Cardiol Heart Vasc. 2018; 21: 7-10, doi: 10.1016/j. ijcha.2018.09.001, indexed in Pubmed: 30211294.

25. Jain H, Chatterjee S, Das TK. Study of platelet count and platelet volume indices in the spectrum of coronary artery diseases and its clinicopathological correlation. Niger J Cardiol. 2018; 15(1): 63.

26. Ateş $\mathrm{O}$, Kiki $\mathrm{I}$, Bilen $\mathrm{H}$, et al. Association of mean platelet volume with the degree of retinopathy in patients with diabetes mellitus. Eur J Gen Med Eur J Gen Med. 2009; 6(2): 99-102, doi: 10.29333/ejgm/82648.

27. Buch A, Kaur S, Nair R, et al. Platelet volume indices as predictive biomarkers for diabetic complications in type 2 diabetic patients. J Lab Physicians. 2017; 9(2): 84-88, doi: 10.4103/09742727.199625, indexed in Pubmed: 28367021. 